\title{
Topological Methods for Set-Valued Nonlinear Analysis
}


This page intentionally left blank 


\section{Topological Methods} for

Set-Valued Nonlinear Analysis

ENAYET U TARAFDAR

The University of Queensland, Australia

\&

MOHAMMAD S R CHOWDHURY

Lahore University of Management Sciences, Pakistan 
Published by

World Scientific Publishing Co. Pte. Ltd.

5 Toh Tuck Link, Singapore 596224

USA office: 27 Warren Street, Suite 401-402, Hackensack, NJ 07601

UK office: 57 Shelton Street, Covent Garden, London WC2H 9HE

\section{British Library Cataloguing-in-Publication Data}

A catalogue record for this book is available from the British Library.

\section{TOPOLOGICAL METHODS FOR SET-VALUED NONLINEAR ANALYSIS}

Copyright (C) 2008 by World Scientific Publishing Co. Pte. Ltd.

All rights reserved. This book, or parts thereof, may not be reproduced in any form or by any means, electronic or mechanical, including photocopying, recording or any information storage and retrieval system now known or to be invented, without written permission from the Publisher.

For photocopying of material in this volume, please pay a copying fee through the Copyright Clearance Center, Inc., 222 Rosewood Drive, Danvers, MA 01923, USA. In this case permission to photocopy is not required from the publisher.

ISBN-13 978-981-270-467-2

ISBN-10 981-270-467-1

Printed in Singapore. 
To

Our beloved professor Dr. Eneyet Ullah Tarafdar, his wife - Mrs. Sangha Mitra, his daughter - Hashina Tarafdar and his two sons Abir Tarafdar and Ashique Tarafdar. 
This page intentionally left blank 


\section{Preface}

This book is a monograph of a significant and recent publications in non-linear analysis involving set-valued mappings. A map $T: X \rightarrow 2^{Y}$ is said to be a setvalued mapping if for each $x \in X, T(x) \subset Y$.

We need analysis, topology and geometry, i.e., a mixture of these three fields, in studying the theory of set-valued mappings. There have been a significant number of publiactions in this area of research over the last 40 years. These have become possible because there are huge applications in the fileds of Physics, Biology, Control Theory, Optimization, Economics and Game Theory.

We shall cover the following topics in this book: contraction mappings, fixed point theorems, minimax inequalities, end points, variational inequalities, generalized variational inequalities, and generalized quasi-variational inequalities, equilibrium analysis in economics, best approximation and fixed point theorems, topological degree theory,and non-expansive types of mappings and fixed point theorems.

In Chapter 5, we shall present variational inequalities, quasi-variational equalities and equilibrium analysis in economics. We have applied the topological methods to study the equilibrium analysis in economics. We shall discuss them in more details in the Introduction Chapter. In Chapter 6, we shall discuss best approximation and fixed point theorems for set-valued mappings in topological vector spaces. Finally, in Chapters 7 and 8 we shall present some aspects of degree theories for set-valued mappings and non-expansive types of mappings and fixed point theorems in locally convex topological vector spaces.

We are very much grateful to Professor Dr. Ken Smith at the Dept. of Mathematics of the University of Queensland for his tremendous help in making this publication possible by compiling the manuscript into Latex format. We are also thankful to Dr. Bevan Thompson of the same department for all his administrative help and encouragement in completing this project.

After the sudden and unfortunate death of Dr. Enayet Tarafdar in November, 2002 , I continued with the project and tried to finish it with the help of my other friends and well wishers who were working in this area of research. In this direction, I would like to mention the names of Dr. George Yuan and Dr. Peter Watson who tried to help me in finishing this project with their valuable suggestons and inputs. 
Thanks goes to Dr. Yuan for partially completing the Promotional Questionnaire of this book before its publication.

Finally, my heartfelt gratitude and thanks goes to Mrs. Sangha Mitra - wife of Dr. Tarafdar, Hashina Tarafdar — daughter of Dr. Tarafdar and his two sons Abir Tarafdar and Ashique Tarafdar and also to my wife - Mrs. Fowzia Akhter. Abir Tarafdar helped in getting the back up of the original soft copy of this project of Dr. Tarafdar. Mrs. Sangha Mitra, Hashina Tarafdar and Ashique Tarafdar helped in searching and collecting some of the documents left at their home which were very helpful for the completion of Dr. Tarafdar's unfinished project. My wife, Fowzia Akhter, helped me invaluably in completing the typing of some chapters of this project.

Lastly, I am very happy to acknowledge that Professor Dr. Zakaullah Khan, the Previous Head of the Dept. of Mathematics of the International Islamic University in Islamabad, Pakistan - my previous work place, helped me so generously that it created an environment for me to complete the proof-reading and final revision of the manuscript of this project for submission to the World Scientific U.K. within the specific deadline of August, 2007.

Mohammad Showkat Rahim Chowdhury Associate Professor

Centre for Advanced Studies in Mathematics Lahore University of Management Sciences (LUMS)

Lahore-54792, Pakistan

October 10, 2007 


\section{Contents}

Preface vii

1. Introduction 1

2. Contraction Mappings 9

2.1 Contraction Mapping Principle in Uniform Topological Spaces and Applications ..................... 9

2.2 Banach Contraction Mapping Principle in Uniform Spaces . . . . . . 10

2.2.1 Successive Approximation . . . . . . . . . . . . . . . . . 14

2.3 Further Generalization of Banach Contraction Mapping Principle . . 27

2.3.1 Fixed Point Theorems for Some Extension of Contraction

Mappings on Uniform Spaces . . . . . . . . . . . . . . . 28

2.3.2 An Interplay Between the Order and Pseudometric Partial

Ordering in Complete Uniform Topological Space . . . . . . . 32

2.4 Changing Norm . . . . . . . . . . . . . . . . . . . . . . . . 34

2.4.1 Changing the Norm . . . . . . . . . . . . . . . 38

2.4 .2 On the Approximate Iteration . . . . . . . . . . . . . . . . . . 43

2.5 The Contraction Mapping Principle Applied to the Cauchy-

Kowalevsky Theorem . . . . . . . . . . . . . . . . . . . 44

2.5.1 Geometric Preliminaries . . . . . . . . . . . . . 45

2.5.2 The Linear Problem . . . . . . . . . . . . . . . . . . . . 46

2.5.3 The Quasilinear Problem . . . . . . . . . . . . . . 50

2.6 An Implicit Function Theorem for a Set of Mappings and Its

Application to Nonlinear Hyperbolic Boundary Value Problem as

Application of Contraction Mapping Principle . . . . . . . . . . 53

2.6.1 An Implicit Function Theorem for a Set of Mappings . . . . . 55

2.6.2 Notations and Preliminaries . . . . . . . . . . . . . . . 60

2.6.3 Results of Smiley on Linear Problem . . . . . . . . . . . . . . 61

2.6.4 Alternative Problem and Approximate Equations . . . . . . . 66 
2.6.5 Application to Nonlinear Wave Equations - A Theorem of Paul Rabinowitz . . . . . . . . . . . . . . . 73

2.7 Set-Valued Contractions . . . . . . . . . . . . . . . . 83

2.7.1 End Points . . . . . . . . . . . . . . . . . . . . 88

2.8 Iterated Function Systems (IFS) and Attractor . . . . . . . . . . . . 91

2.8.1 Applications . . . . . . . . . . . . . . . . . 94

2.9 Large Contractions . . . . . . . . . . . . . . . . . . . . 103

2.9.1 Large Contractions . . . . . . . . . . . . . . . . . . . . . . 104

2.9.2 The Transformation . . . . . . . . . . . . . . . . 105

2.9.3 An Existence Theorem . . . . . . . . . . . . . . . . . 106

2.10 Random Fixed Point and Set-Valued Random Contraction . . . . . . 107

3. Some Fixed Point Theorems in Partially Ordered Sets 113

3.1 Fixed Point Theorems and Applications to Economics . . . . . . . . 113

3.2 Fixed Point Theorem on Partially Ordered Sets . . . . . . . . . . . . 113

3.3 Applications to Games and Economics . . . . . . . . . . . . . . 116

3.3 .1 Game ..................... 117

3.3 .2 Economy. . . . . . . . . . . . . . . . . 118

3.3.3 Pareto Optimum . . . . . . . . . . . . . . . . . 119

3.3.4 The Contraction Mapping Principle in Uniform Space via Kleene's Fixed Point Theorem . . . . . . . . . . . . . . . 120

3.3.5 Applications on Double Ranked Sequence . . . . . . . . . . . 124

3.4 Lattice Theoretical Fixed Point Theorems of Tarski . . . . . . . . . 125

3.5 Applications of Lattice Fixed Point Theorem of Tarski to Integral Equations . . . . . . . . . . . . . . . . . . 131

3.6 The Tarski-Kantorovitch Principle . . . . . . . . . . . . . . . . . . 134

3.7 The Iterated Function Systems on $\left(2^{X}, \supset\right) \ldots$. . . . . . . . . . 136

3.8 The Iterated Function Systems on $(C(X), \supset)$. . . . . . . . . . . . 139

3.9 The Iterated Function System on $(K(X), \supset)$. . . . . . . . . . . . . 141

3.10 Continuity of Maps on Countably Compact and Sequential Spaces . 142

3.11 Solutions of Impulsive Differential Equations . . . . . . . . . . . . . 146

3.11.1 A Comparison Result . . . . . . . . . . . . . . . . . . . . . 147

3.11 .2 Periodic Solutions . . . . . . . . . . . . . . . . . . . . 149

4. Topological Fixed Point Theorems 151

4.1 Brouwer Fixed Point Theorem . . . . . . . . . . . . . . . . 151

4.1.1 Schauder Projection . . . . . . . . . . . . . . . . 160

4.1.2 Fixed Point Theorems of Set Valued Mappings with

Applications in Abstract Economy . . . . . . . . . . . . . 162

4.1.3 Applications . . . . . . . . . . . . . . . . . . . 167

4.1.4 Equilibrium Point of Abstract Economy . . . . . . . . . . . 169

4.2 Fixed Point Theorems and KKM Theorems . . . . . . . . . . . . . . 171 
4.2.1 Duality in Fixed Point Theory of Set Valued Mappings . . . . 174

4.3 Applications on Minimax Principles . . . . . . . . . . . . . . . . 177

4.3.1 Applications on Sets with Convex Sections . . . . . . . . . . . 179

4.4 More on Sets with Convex Sections . . . . . . . . . . . . . . . . . . . 182

4.5 More on the Extension of KKM Theorem and Ky Fan's Minimax Principle. . . . . . . . . . . . . . . . . . . . 190

4.6 A Fixed Point Theorem Equivalent to the Fan-Knaster-KuratowskiMazurkiewicz Theorem . . . . . . . . . . . . . . . . . . . 195

4.7 More on Fixed Point Theorems . . . . . . . . . . . . . . . . . . . . 200

4.8 Applications of Fixed Point Theorems to Equilibrium Analysis in Mathematical Economics and Game Theory . . . . . . . . . . . . . . 206

4.8.1 Fixed Point and Equilibrium Point . . . . . . . . . . . . . . 207

4.8.2 Existence of Maximal Elements . . . . . . . . . . . . . . . 211

4.8.3 Equilibrium Existence Theorems . . . . . . . . . . . . . . . . 213

4.9 Fixed Point of $\psi$-Condensing Mapping, Maximal Elements and Equilibria . . . . . . . . . . . . . . . . . . . . . 224 4.9.1 Equilibrium on Paracompact Spaces . . . . . . . . . . . . . 237

4.9.2 Equilibria of Generalized Games . . . . . . . . . . . . . . . . 240

4.9 .3 Applications .. . . . . . . . . . . . . . . . . . 243

4.10 Coincidence Points and Related Results, an Analysis on $H$-Spaces . 244

4.11 Applications to Mathematical Economics: An Analogue of Debreu's Social Equilibrium Existence Theorem . . . . . . . . . . . . . 261

5. Variational and Quasivariational Inequalities in Topological

Vector Spaces and Generalized Games

5.1 Simultaneous Variational Inequalities . . . . . . . . . . . . . . . . 265

5.1.1 Variational Inequalities for Single Valued Functions . . . . . . 265

5.1.2 Solutions of Simultaneous Nonlinear Variational Inequalities . 268

5.1.3 Application to Nonlinear Boundary Value Problem for Quasilinear Operator of Order $2 \mathrm{~m}$ in Generalized Divergence Form 276

5.1.4 Minimization Problems and Related Results . . . . . . . . . . 280

5.1.5 Extension of a Karamardian Theorem . . . . . . . . . . . . . 282

5.2 Variational Inequalities for Setvalued Mappings . . . . . . . . . . . . 284

5.2 .1 Simultaneous Variational Inequalities . . . . . . . . . . . . . . 287

5.2.2 Implicit Variational Inequalities — The Monotone Case . . . 292

5.2.3 Implicit Variational Inequalities — The USC Case . . . . . . 296

5.3 Variational Inequalities and Applications . . . . . . . . . . . . . . 301

5.3.1 Application to Minimization Problems . . . . . . . . . . . . . 304

5.4 Duality in Variational Inequalities . . . . . . . . . . . . . . 306

5.4.1 Some Auxiliary Results . . . . . . . . . . . . . . . . . . 309

5.5 A Variational Inequality in Non-Compact Sets with Some

Applications . . . . . . . . . . . . . . . . . 312 
5.6 Browder-Hartman-Stampacchia Variational Inequalities for Set-Valued Monotone Operators . . . . . . . . . . . . . . . . . . 321

5.6.1 A Minimax Inequality . . . . . . . . . . . . . . . . . . 321

5.6.2 An Existence Theorem of Variational Inequalities . . . . . . . 322

5.7 Some Generalized Variational Inequalities with Their Applications . 325

5.7.1 Some Generalized Variational Inequalities . . . . . . . . . . . 325

5.7.2 Applications to Minimization Problems . . . . . . . . . . . . . 333

5.8 Some Results of Tarafdar and Yuan on Generalized Variational

Inequalities in Locally Convex Topological Vector Spaces . . . . . . . 335

5.8.1 Some Generalized Variational Inequalities . . . . . . . . . . . 337

5.9 Generalized Variational Inequalities for Quasi-Monotone and QuasiSemi-Monotone Operators . . . . . . . . . . . . . . . . . . . . 340

5.9.1 Generalization of Ky Fan's Minimax Inequality . . . . . . . . 346

5.9 .2 Generalized Variational Inequalities . . . . . . . . . . . . . . . 348

5.9.3 Fixed Point Theorems . . . . . . . . . . . . . . . . . . 358

5.10 Generalization of Ky Fan's Minimax Inequality with Applications to Generalized Variational Inequalities for Pseudo-Monotone Type I Operators and Fixed Point Theorems . . . . . . . . . . . . . . 363

5.10.1 Generalization of Ky Fan's Minimax Inequality . . . . . . . . 365

5.10 .2 Generalized Variational Inequalities . . . . . . . . . . . . . . . 372

5.10 .3 Applications to Fixed Point Theorems . . . . . . . . . . . . . 377

5.11 Generalized Variational-Like Inequalities for Pseudo-Monotone

Type I Operators . . . . . . . . . . . . . . . . . . . . 379

5.11 .1 Existence Theorems for $G V L I(T, \eta, h, X, F) \ldots 383$

5.12 Generalized Quasi-Variational Inequalities . . . . . . . . . . . . 388

5.12.1 Generalized Quasi-Variational Inequalities for Monotone and Lower Semi-Continuous Mappings . . . . . . . . . . . . . . 388

5.12.2 Generalized Quasi-Variational Inequalities for Upper SemiContinuous Mappings Without Monotonicity . . . . . . . . . 393

5.13 Generalized Quasi-Variational Inequalities for Lower and Upper Hemi-Continuous Operators on Non-Compact Sets . . . . . . . . . . 397

5.13.1 Generalized Quasi-Variational Inequalities for Lower HemiContinuous Operators . . . . . . . . . . . . . . 398

5.13.2 Generalized Quasi-Variational Inequalities for Upper HemiContinuous Operators . . . . . . . . . . . . . . . . . 404

5.14 Generalized Quasi-Variational Inequalities for Upper Semi-

Continuous Operators on Non-Compact Sets . . . . . . . . . . . . . 409

5.14.1 Non-Compact Generalized Quasi-Variational Inequalities . . . 410

5.15 Generalized Quasi-Variational Inequalities for Pseudo-Monotone

Set-Valued Mappings . . . . . . . . . . . . . . . . . . . . . 415

5.15.1 Generalized Quasi-Variational Inequalities for Strong PseudoMonotone Operators . . . . . . . . . . . . . . 415 
5.15.2 Generalized Quasi-Variational Inequalities for PseudoMonotone Set-Valued Mappings . . . . . . . . . . . . . . . . . 421

5.16 Non-Linear Variational Inequalities and the Existence of Equilibrium in Economics with a Riesz Space of Commodities . . . . . . . . . . . 426

5.16.1 Existence of Equilibrium Lemma . . . . . . . . . . . . . . . . 428

5.17 Equilibria of Non-compact Generalized Games with $\mathcal{L}^{*}$

Majorized Preference Correspondences . . . . . . . . . . . . . . . . 430

5.17 .1 Existence of Maximal Elements . . . . . . . . . . . . . . . . 430

5.17.2 Existence of Equilibrium for Non-Compact Abstract Economies . . . . . . . . . . . . . . . . 434

5.18 Equilibria of Non-Compact Generalized Games . . . . . . . . . . . . 438

5.18 .1 Equilibria of Generalized Games . . . . . . . . . . . . . . . . 442

5.18.2 Tarafdar and Yuan's Application on Existence Theorem of Equilibria for Constrained Games . . . . . . . . . . . . . . 445

6. Best Approximation and Fixed Point Theorems for Set-Valued Mappings in Topological Vector Spaces

6.1 Single-Valued Case . . . . . . . . . . . . . . . . . . . . . . . . 448

6.2 Set-Valued Case . . . . . . . . . . . . . . . . . . . 452

6.2.1 Some Lemmas and Relevant Results . . . . . . . . . . . . . . 454

7. Degree Theories for Set-Valued Mappings 463

7.1 Degree Theory for Set-Valued Ultimately Compact Vector Fields . . 463 7.1.1 Properties of the Degree of Ultimately Compact Vector Fields 465 $7.1 .2 k$ - $\phi$-Contractive Set Valued Mappings . . . . . . . . . . . . . 467

7.2 Coincidence Degree for Non-Linear Single-Valued Perturbations of Linear Fredholm Mappings . . . . . . . . . . . . . . . . . . . . . 471

7.2.1 An Equivalence Theorem . . . . . . . . . . . . . . . . . . 473

7.2.2 Definition of Coincidence Degree . . . . . . . . . . . . . . . . 474

7.2.3 Properties of the Coincidence Degree . . . . . . . . . . . . . . 475

7.3 On the Existence of Solutions of the Equation $L x \in N x$ and a Coincidence Degree Theory . . . . . . . . . . . . . . . . . . . 478

7.3.1 Coincidence Degree for Set-Valued $k-\phi$-Contractive Perturbations of Linear Fredholm Mappings . . . . . . . . . . . . . . 479

7.4 Coincidence Degree for Multi-Valued Mappings with Non-Negative Index . . . . . . . . . . . . . . . . . . . . . . . . 497

7.4.1 Basic Assumptions and Main Results in Akashi (1988) . . . . 497

7.4.2 Akashi's Basic Properties of Coincidence Degree . . . . . . . . 502

7.4.3 Application to Multitivalued Boundary Value Problem for Elliptic Partial Differential Equation . . . . . . . . . . . . 503

7.5 Applications of Equivalence Theorems with Single-Valued Mappings: An Approach to Non-Linear Elliptic Boundary Value Problems . . . 507 
7.5.1 Tarafdar's Application to Elliptic Boundary Value Problems . 521

7.6 Further Results in Coincidence Degree Theory . . . . . . . . . . . . 525

7.7 Tarafdar and Thompson's Theory of Bifurcation for the Solutions of

Equations Involving Set-Valued Mapping . . . . . . . . . . . . 528

7.7.1 Characteristic Value and Multiplicity . . . . . . . . . . . . 532

7.7.2 Tarafdar and Thompson's Results on the Theory of Bifurcation .................. . . 532

7.7.3 Tarafdar and Thompson's Application on the Theory of Bifurcation . . . . . . . . . . . . . . . . 539

7.8 Tarafdar and Thompson's Results on the Solvability of Non-Linear and Non-Compact Operator Equations . . . . . . . . . . . . . . . . . 542

7.8.1 Measure of Noncompactness and Set Contraction . . . . . . . 542

7.8 .2 Epi Mappings . . . . . . . . . . . . . . . . . . . . . 546

7.8.3 Tarafdar and Thompson's $(p, k)$-Epi Mappings on the Whole Space . . . . . . . . . . . . . . . . . 555

7.8.4 Tarafdar and Thompson's Applications of $(p, k)$-Epi Mappings in Differential Equations . . . . . . . . . . . . . . 556

8. Nonexpansive Types of Mappings and Fixed Point Theorems in Locally Convex Topological Vector Spaces

8.1 Nonexpansive Types of Mappings in Locally Convex Topological Vector Spaces . . . . . . . . . . . . . . . . . 563

8.1.1 Nonexpansive Mappings . . . . . . . . . . . . . . . . . . 563

8.2 Set-Valued Mappings of Nonexpansive Type . . . . . . . . . . . . . . 571

8.2.1 Normal Structure and Fixed Point Theorems . . . . . . . . . 572

8.2.2 Another Definition of Nonexpansive Set-Valued Mapping and Corresponding Results on Fixed Point Theorems . . . . . 575

8.3 Fixed Point Theorems for Condensing Set-Valued Mappings on Locally Convex Topological Vector Spaces . . . . . . . . . . . . . . . 576

8.3.1 Measure of Precompactness and Non-Precompactness . . . . . 577

8.3.2 Condensing Mappings . . . . . . . . . . . . . . . . 578

8.3.3 Fixed Point Theorems . . . . . . . . . . . . . . . . . 580

$\begin{array}{ll}\text { Bibliography } & 583\end{array}$

Index 